\title{
Melhores Práticas em Gerenciamento de Projetos e.Xactal CIVIS PELO USO DO BUILDING INFORMATION MODELING - BIM
}

ISSN: 1984-3151

\author{
Best Practices in Civil Project Management by the Use of \\ BUILDING INFORMATION MODELING - BIM
}

Ciro Jr. Martins Ribeiroㅁ; Romante Ezer Ferreira Rodrigues ${ }^{2}$

1 Pós-graduado em Gestão de Projetos de Engenharia. Centro Universitário de Belo Horizonte - UniBH, 2016. Belo Horizonte-MG. ciromartinsara@gmail.com.

2 Mestre em Administração com Ênfase em Finanças. Universidade Federal de Minas Gerais - UFMG, 2014. Professor do Centro Universitário de Belo Horizonte UniBH. Belo Horizonte-MG. romantebh@gmail.com.

Recebido em 14/06/2017; Publicado em 23/06/2019

RESUMO: Na contemporaneidade, as falhas mais comuns nos processos de projetos civis estão nos erros ou inexistência da gestão. Este artigo explicita como os softwares de plataforma BIM (Building Information Modeling) podem ser uma solução para processos de projetos mais eficientes e integrados com o gerenciamento e tem como objetivo demonstrar se o uso da plataforma pode ser uma solução para sérios problemas de desenvolvimento eficiente de projetos (design). Através de pesquisa bibliográfica foi possível identificar o potencial do BIM, compará-lo com outros tipos de softwares e identificar a tendência da gestão como fator essencial nos projetos civis atuais, concluindo positivamente uma forte relação entre o problema levantado e a solução estudada.

PALAVRAS-CHAVE: Melhores práticas. Construção civil. Projetos. BIM.

ABSTRACT: In contemporary times, the most common failures in civil project processes are in lack or inexistence of management. This article explains how BIM (Building Information Modeling) softwares can be a solution for more efficient and integrated project processes with management and aims to demonstrate if the use of the platform can be a solution to serious problems of efficient development of designs. Through bibliographic research it was possible to identify the potential of BIM, compare it with other types of software and identify the tendency of management as an essential factor in current civil projects, concluding positively a Strong relation between the problem raised and a solution studied.

KEYWORDS: Best practices. Civil construction. Design. BIM.

\section{INTRODUÇÃo}

A Gestão de Projetos Civis, assim como todas as áreas de negócios, carece cada vez mais de aprimoramento, no condizente à utilização de novos conhecimentos e ferramentas que colaborem no sentido da objetividade dos resultados relativos ao uso dos recursos versus escopo acordado, cumprimento dos cronogramas e a gestão do conhecimento, como base para 0 aperfeiçoamento contínuo destas práticas. Após observação de alguns casos práticos no ambiente desse mercado, identificaram-se diversas 
falhas nos seus processos, muitas vezes pelo desconhecimento de ferramentas disponíveis para o gerenciamento e desenvolvimento de projetos. A tecnologia contemporânea disponibiliza ferramentas de uso específico para a construção civil, com base nas melhores práticas estudadas e por opção, pela completude de seus relatórios e detalhamentos e, por toda a abrangência alcançada, uso de softwares de plataforma BIM (Building Information Modeling) para os escritórios e empresas de projetos civis apresentase como excelente alternativa para sanar a falta de qualidade dos projetos, a ineficiência dos processos de desenvolvimento e os problemas de gestão detectados no setor. Tal questão é tratada na atualidade como uma grande tendência, com promessa de domínio tecnológico.

\section{OBJetivos}

Demonstrar como o uso de softwares de plataforma BIM para o mercado de projetos civis pode ser uma solução para sérios problemas de desenvolvimento eficiente de projetos (design). Será desenvolvido de forma a conceituar e identificar o potencial da plataforma BIM; comparar a plataforma com as outras usuais no mercado de projetos civis; conceituar a Gestão de Projetos e identificar sua tendência contemporânea como fator essencial nos projetos civis; e atrelar as deficiências mais comuns da gestão de projetos com qualidades da plataforma BIM de modo a apresentar as soluções.

\section{Metodologia}

Pesquisa bibliográfica feita a partir de análises teóricas já realizadas e publicadas (artigos científicos, livros e dissertações). Apesar de em maioria, as investigações científicas já iniciarem com tais explorações, existem pesquisas que se apoiam unicamente em pesquisas bibliográficas (como é o caso desta) que permite recolher informações e embasamentos teóricos sobre o tema, e respectivas soluções para a problemática proposta (FONSECA, 2002).

Tal método permite, por investigação detalhada, o acesso a fatos e fenômenos anteriormente pesquisados e relatados (ROBSON, 2002), e oferece como principal vantagem a ampliação da gama de conhecimento obtido para os estudos pretendidos, uma vez que permite acesso a mais dados do que se pesquisaria de forma direta (GIL, 2002).

O uso dessa metodologia possibilitará agrupar um número de informações usualmente encontradas de forma desconectada, levando a compreender melhor como o uso de tais informações pode sanar os problemas levantados.

\section{DesenVolvimento}

Desde a década de 1970 têm-se aperfeiçoados os conceitos de processo de mesma origem da plataforma de softwares BIM (Building Information Modeling ou Modelagem da Informação da Construção) (YESSIOS, 2004), que nos últimos tempos tem tomado mais espaço no mercado de projetos e processos de construções civis (EASTMAN et al., 2008).

Os softwares BIM têm substituído no setor de projetos civis, os softwares mais usados; e trazem uma forma de sistematização completamente distinta para 0 ato de projetar.

O Desenho Assistido por Computador (CAD), com desenvolvimento inicial datado em 1960, tem sido a plataforma de representação e desenvolvimento de projetos civis mais utilizada em uso comercial, desde a década de 1980 (CASTELAN; MILANEZ; FRITZEN, 2016; MARQUES; FLORES; SOUTO, 2017). Como exemplo, tem-se atualmente, como softwares mais 
comuns para a construção civil, o Autodesk AutoCAD e o Trimble Sketchup.

Esclarece-se desde já, que o presente trabalho utiliza o termo "CAD" para se referir aos softwares utilizados anteriormente à criação da plataforma BIM, que também é uma plataforma de "desenho assistido por computador", porém com características de programação paramétrica que ultrapassam a representação de desenhos e modelagens tridimensionais.

O projeto pode ser concebido em software BIM considerando todas as suas etapas. Desde as definições totais de projeto (design), até planejamento, orçamentos e o suporte para os próprios profissionais de execução. O modelo é feito através de processo paramétrico em que os elementos criados são os elementos da construção, e não somente elementos gráficos para formar o desenho ou modelagem como são feitos em CAD, seja em representação bidimensional ou tridimensional.

Os programas que utilizam tal plataforma têm evoluído exponencialmente suas ferramentas e hoje conseguem trazer, além da visão mais completa do processo para o projetista, condições de conectar os projetos de diferentes disciplinas, potencializando a eficiência do processo de compatibilização (CATELANI, 2016).

Simultaneamente a tal inovação, ocorre outro fenômeno no mercado de projetos, aparentemente desconexo ao anterior, que é a necessidade de melhorias no gerenciamento dos projetos civis.

O gerenciamento de projetos tem sido estudado por dois pontos de vista: um considerando melhorar as práticas de projeto e outro incluindo o projetista em todos os processos em que o projeto se envolve. A qualidade dos projetos e o aumento do potencial das empresas têm sido cada vez mais relacionados aos métodos organizacionais usados (TZORTZOPOULOS; COOPER, 2007).
Ambos os pontos de vista fazem crer que num mercado tão competitivo e com facilidade de fluidez da informação, necessita-se cada vez mais de gestores de projetos e que tais gestores tenham o conhecimento pleno de todo o processo de concepção, elaboração, desenvolvimento e administração do projeto.

É na interseção desses dois assuntos que se fazem as disposições deste artigo.

\subsection{CONCEITUAÇÃo do BIM E IDENTIFICAÇÃo dE SEU POTENCIAL}

O American Institute of Architects (AIA) define BIM como "uma tecnologia baseada em um modelo que está associada a um banco de dados de informações sobre um projeto" (CATELANI, 2016).

Tal modelo tem como intenção ser uma base sólida de dados para a criação e disponibilização para consultas durante o processo de projeto ou para decisões futuras.

Além de aumentar a quantidade e a precisão das informações durante o projeto, o modelo permite que tais informações melhore a probabilidade de acerto nas decisões tomadas, uma vez que todos os colaboradores podem ver e analisar com mais facilidade os impactos destas decisões no projeto.

Para o modelo produzido em BIM, existem diferenciações de referências de dimensões do modelo (3D, 4D, 5D, 6D) e elas se dão de acordo com os tipos de fornecimento de informações. Os modelos ditos 3D, contêm informações espaciais e físicas de elementos construção projetados. Os modelos 4D são os que além das informações físicas de projeto, possuem informações de tempo, ordem e produtividade da construção. Os modelos 5D possuem dados de valores de custo - mão-de-obra, maquinário, equipamentos e demais despesas da construção. Os 
modelos $6 \mathrm{D}$ são os que, além de todas a informações sobre o projeto e construção do edifício, possuem dados sobre o uso, como durabilidade de materiais, eficiência energética, manutenção.

Com modelos computacionais de tamanha competência, consegue-se realizar projetos de alta eficiência e com compatibilização simulada de forma a garantir a qualidade do produto final, tal como facilitar todos os processos administrativos que acompanham a criação (COMPESTRINI et al., 2015).

\subsection{COMPARATIVO DO BIM COM O CAD}

Nos sistemas CAD, a representação do projeto se dá pela criação de elementos geométricos utilizando coordenadas no plano do software. O desenho é uma junção de tais elementos de forma a gerar uma representação meramente ilustrativa dos elementos construtivos.

Modificações em um projeto desenvolvido com tal sistema implicam em alterações manuais nos desenhos, posteriormente a uma busca meticulosa pelas intervenções da modificação feita em outras representações (COELHO; MATTAR; NOVAES, 2006). O processo tradicional de desenvolvimento de projetos é uma forma mais simples de trabalho, em que $o$ projeto desenvolvido somente possui informações de desenho somado a documentos produzidos externamente.

Em completa evolução do sistema tradicional, o BIM adota modelos paramétricos e trata os elementos construtivos como sua real forma, permitindo obtenção de informações de materiais, técnicas construtivas e determinação das aplicações. As modificações de um projeto desenvolvido em BIM são feitas em uma única decisão no modelo de trabalho e permite plena compreensão das interferências com outros dados, dimensões e disciplinas contidas no projeto (CATELANI, 2016).
Além dos dados físicos e espaciais (em formato de desenho e maquete eletrônica) em configurações melhoradas, o modelo abrange indicadores geográficos e de influência natural, tabelas e processos de operação da construção - tudo no mesmo arquivo e modelo (FERREIRA, 2007).

O BIM também se mostra vantajoso em possuir ferramentas para as práticas de engenharia simultânea e trabalho colaborativo, possibilitando sanar tanto os problemas de logística de trabalho em grandes equipes, nas quais os prazos são desafiadores, quanto os problemas de conexão e propagação de informações entre as diferentes disciplinas (CATELANI, 2016).

\subsection{Conceituação da Gestão de Projetos e IDENTIFICAÇÃO DE SUA TENDÊNCIA COMO FATOR Essencial nos Projetos Civis}

O PMI (Project Management Institute) define gestão de projetos como o processo pelo qual se aplicam conhecimentos, capacidades, instrumentos e técnicas às atividades do projeto de forma a satisfazer as necessidades e expectativas dos vários stakeholders, que são indivíduos ativamente envolvidos no projeto ou cujo resultado deste poderá afetá-los positivamente ou negativamente, ou seja, é um agrupamento de conceitos e técnicas aplicadas estrategicamente a fim de liderar equipes e controlar o projeto para obtenção de um produto final (CAMPOS, 2012).

Nos anos 90, as empresas começaram a conviver com um mercado mais severo, as pressões competitivas criaram a necessidade de criar produtos de maior qualidade e com prazos mais curtos; juntamente à preocupação de manter as relações de longo prazo com os clientes.

Para sobreviver nesse mercado cada vez mais intrínseco a essas características, a gestão de projetos garante a permanência da empresa através 
do planejamento e controle de uma série de atividades integradas (KERZNER, 2002).

O gerenciamento dos projetos de uma empresa hoje é aplicado estrategicamente para criar um aumento de perspectivas de mercado, e não somente para acompanhar projetos isolados com necessidade de supervisão - como era tratado anteriormente. Os projetos acontecendo de forma simultânea e o quadro de trabalhos em constante mudança cria organismos dinâmicos dentro da empresa (DINSMORE, 1999).

O gerente de projetos atualmente ganha destaque dentro das organizações pela evolução e relevância do gerenciamento de projetos. A profissão de gerenciamento de projetos é emergente e bastante promissora (COELHO P., 2005, p.29).

\subsection{Soluções para Gestão de Projetos de Construção CiVIL AtravÉs do BIM}

Com intenção de facilitar as relações de problemas e soluções, o conteúdo a seguir será dividido em fases de projeto (planejamento, execução e conclusão), em que cada título terá uma breve descrição do conceito da fase e dos problemas causados pelos softwares e processos convencionais atuais. Posteriormente, as soluções e vantagens que o uso da plataforma BIM pode oferecer.

\subsubsection{Fase de Planejamento}

A fase de Planejamento de um projeto preocupa-se em estruturar toda a operação e analisar a viabilidade sua. Ela detalha o escopo e se preocupa com o custo, prazo e qualidade. Partes dessa operação são o detalhamento das atividades e a organização das divisões de trabalho; análise dos prazos e programação das atividades em tempo hábil para atender os mesmos; formação da equipe; estruturação do sistema de comunicação (MENEZES, 2001).

$\mathrm{Na}$ estruturação dos processos de projeto a necessidade se volta em maioria para as análises de documentos iniciais, que tratam de orçamentos, escopo e contratações (CAMPOS, 2012). Apesar de tal etapa depender de forma mais geral de documentos de texto e planilhas, futuramente na fase de execução tais informações (principalmente as de escopo) deveram ser atreladas aos processos de design. Os processos desenvolvidos com o uso do $C A D$ - plataforma ainda mais utilizada hoje, por se tratar de um sistema no qaul os elementos não possuem informações inerentes ao projeto, fazem com que este atrelamento de informações futuras seja feito de forma manual e pouco eficiente (FLORIO, 2007).

\subsubsection{FASE DE EXECUÇÃo}

$\mathrm{Na}$ fase de Execução de um projeto acontecem os processos de confecção do produto final. É o trabalho de execução propriamente dito. Partes dessa etapa são aplicação prática das estratégias definidas na fase de planejamento; ativação do plano de comunicação definido; efetuar reprogramações do projeto na medida do necessário; controlar o desempenho de qualidade geral dos processos; administração de contratos; gerenciamento de pessoas envolvidas (MENEZES, 2001).

Para essa etapa, a escolha das ferramentas de elaboração e desenvolvimento do design são de extrema importância para a eficiência do processo global. Os problemas enfrentados na execução do projeto estão de forma generalizada nos processos de confecção do design (desenhos técnicos e representações formais) atrelados às deficiências de comunicação entre as partes envolvidas (FLORIO, 2007), como mostra a Figura 1. Na plataforma CAD, como já mencionado anteriormente, tais processos de confecção e modificações são feitos de forma completamente manual e a comunicação se dá por meios externos à plataforma em que o design está sendo trabalhado. Para o gerente de projetos, é a fase 
na qual se controla e corrige (DINSMORE; CAVALIERI, 2003).

Figura 1 - Interação entre as disciplinas nos processos de projeto baseados em documentos (CAD) (superior). Modelo compartilhado, como é nos projetos em que se utiliza o BIM (inferior)
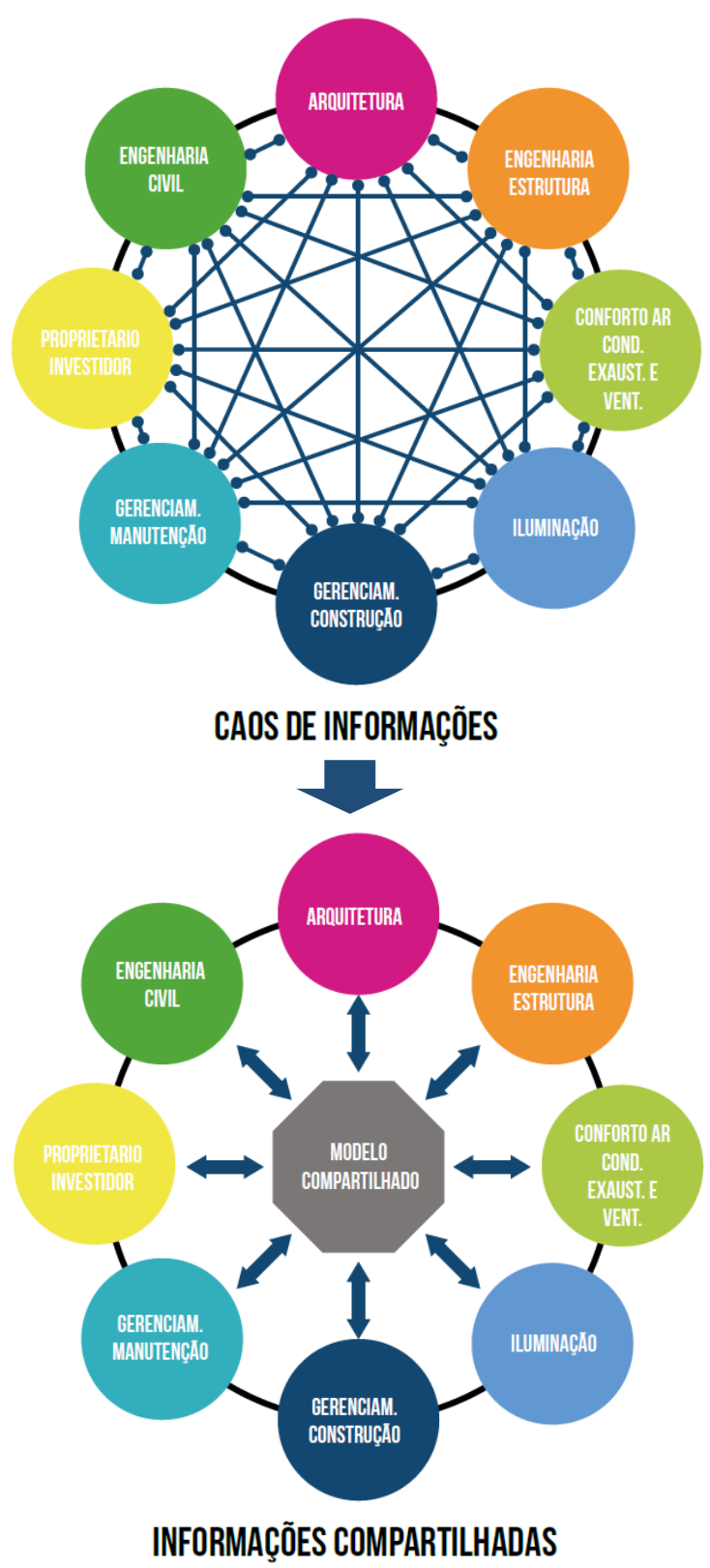

Fonte - CATELANI, 2016.

\subsubsection{FASE de Conclusão}

Última fase do projeto, a Conclusão é o término do processo global. Tem como objetivos o desligamento ou realocação gradual dos envolvidos; finalização dos documentos de projeto; relatórios de lições aprendidas; avaliações de desempenho; encerramento dos contratos; entrega do produto final (MENEZES, 2001).

Para o encerramento, a documentação de registros a ser gerada depende diretamente dos relatórios e análises do decorrer da fase de execução (CAMPOS, 2012). Nos sistemas convencionais atuais, tais registros são feitos de forma temporária em anotações nas próprias pranchas de desenho ou com documentos externos à plataforma do design, ou até mesmo inexistem (COELHO; MATTAR; NOVAES., 2006).

\subsubsection{Soluções Apresentadas Através do Uso Do BIM}

Como explicitado anteriormente na explicação sobre as referências de dimensões do modelo (3D, 4D, 5D E 6D), o BIM possui a capacidade de gerar documentações sobre o modelo em desenvolvimento, abrangendo detalhes de tempo e custo. Tais ferramentas permitem de forma completa 0 atrelamento ao design das informações necessárias à etapa de planejamento do projeto, passando os orçamentos, divisões de trabalho, etc. (COELHO; MATTAR; NOVAES, 2006).

Tal como mostrado na Figura 1, as melhorias no quesito comunicação começam pela forma de trabalho integrada pelo modelo. O BIM contribui de forma a unir todas as informações junto ao modelo, formando um processo colaborativo entre todos os participantes do desenvolvimento. Tal formato de comunicação facilita as edições no design, as compatibilizações entre as disciplinas e a difusão das informações globais do projeto. O processo que anteriormente era hierárquico se torna colaborativo (FLORIO, 2007).

A substituição do processo de confecção das representações feitas com elementos geométricos 
sem informações de construção atribuídas por simulações dos elementos construtivos reais representa a maior evolução da troca de plataformas (CAD-BIM). Os dados do projeto antes não computáveis, no BIM são fornecidos junto aos demais aspectos do modelo (BERNSTEIN, 2004).

De acordo com Seletsky (2006), o BIM permite a melhor análise crítica dos projetos por oferecer melhores condições de avaliação.

Além das vantagens de eficiência no processo de projeto, a utilização do BIM tem trazido para as empresas melhor retorno financeiro sobre 0 investimento (ROI). Tal como retrata Assaf (2010), o ROI é a relação entre a quantidade de lucro (ou prejuízo) e os ativos da empresa.

A empresa McGraw Hill Construction fez uma pesquisa sobre os retornos do uso do BIM com empreiteiras de 9 países e gerou, dentre outros, os relatórios gráficos, como mostram as Figuras 2 e 3.

Figura 2 - Retorno de investimento percebido pelas empreiteiras utilizadoras do BIM

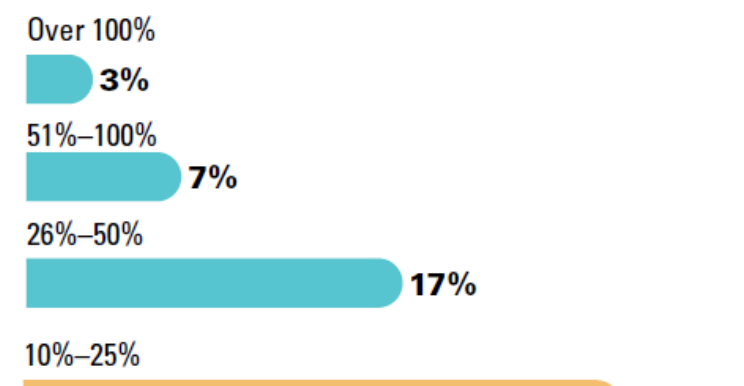

$27 \%$

Less than $10 \%$

$20 \%$

Break-Even

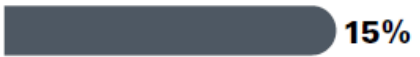

Negative

$10 \%$

ROI muito positivo (azul); ROI positivo moderado (laranja); ROI negativo ou break-even (cinza).

Fonte - MCGRAW HILL CONSTRUCTION, 2014.
Figura 3 - Influência que o nível de engajamento da empreiteira no uso do BIM tem na percepção do ROI

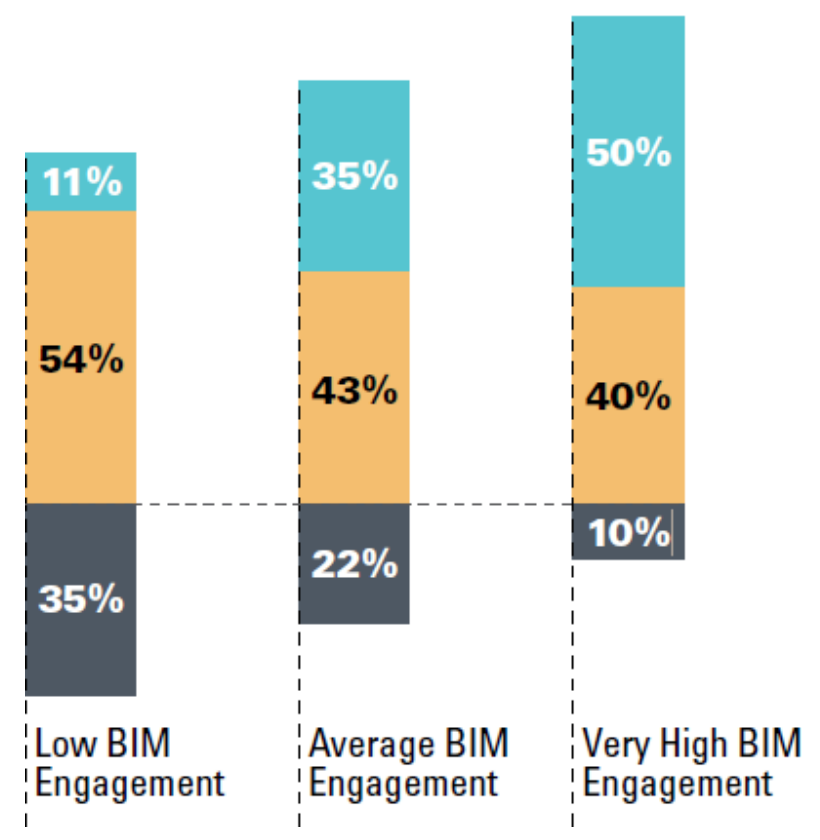

ROI muito positivo (azul) - acima de $25 \%$; ROI positivo moderado (laranja) - 1 a $25 \%$; ROI negativo ou breakeven (cinza).

Fonte - MCGRAW HILL CONSTRUCTION, 2014.

\subsection{ANÁLISE DE DADOS}

Analisando os dados obtidos no Referencial Teórico, pode-se perceber que a profissão do gerente de projetos está em ascensão no mercado contemporâneo de projetos civis e sua devida importância está sendo mais bem reconhecida atualmente. A gestão de projetos vai além da administração dos honorários e prazos, se estendendo até a coordenação de recursos humanos e a qualidade dos processos de design.

Os processos convencionais e mais usados atualmente - CAD para o design somado aos documentos gerados de forma externa apresentam ferramentas ultrapassadas em comparação com 0 BIM. A utilização de um modelo único que concentra informações diversas e de forma completa consegue atingir um alto grau de eficiência nos processos e 
comunicação entre equipes e disciplinas. Tal como evidenciado na Tabela 1 , as diferenças entre as plataformas são claras e podem ser o diferencial procurado para o mercado competitivo atual.

Tabela 1 - Comparativo funcional entre competências principais do processo de design nas plataformas CAD e BIM

\begin{tabular}{|c|c|c|}
\hline \multirow{2}{*}{ Competências } & \multicolumn{2}{|c|}{ Comparativo de características } \\
\hline & CAD & BIM \\
\hline Criação formal & $\begin{array}{l}\text { Uso de elementos } \\
\text { geométricos; } \\
\text { desenhos ou } \\
\text { modelagens } \\
\text { meramente } \\
\text { ilustrativas }\end{array}$ & $\begin{array}{l}\text { Uso de elementos } \\
\text { paramétricos; } \\
\text { simulação de } \\
\text { elementos } \\
\text { construtivos com } \\
\text { propriedades } \\
\text { reais }\end{array}$ \\
\hline Produto formal & $\begin{array}{l}\text { Desenho ou } \\
\text { modelagem } \\
\text { simples; produto } \\
\text { proporcional ao } \\
\text { criado na } \\
\text { dimensão } \\
\text { trabalhada }\end{array}$ & $\begin{array}{c}\text { Modelo } \\
\text { tridimensional } \\
\text { parametrizado de } \\
\text { acordo com o } \\
\text { elemento criado }\end{array}$ \\
\hline $\begin{array}{l}\text { Indicadores, } \\
\text { tabelas e } \\
\text { gráficos }\end{array}$ & $\begin{array}{c}\text { Não possui; } \\
\text { possibilidade de } \\
\text { confecção manual }\end{array}$ & $\begin{array}{c}\text { Possui todos já } \\
\text { integrado ao } \\
\text { modelo }\end{array}$ \\
\hline $\begin{array}{l}\text { Trabalho } \\
\text { colaborativo/ } \\
\text { simultâneo }\end{array}$ & $\begin{array}{l}\text { Não possui; sem } \\
\text { possibilidade. }\end{array}$ & $\begin{array}{l}\text { Possível por } \\
\text { sistema já } \\
\text { integrado aos } \\
\text { softwares }\end{array}$ \\
\hline
\end{tabular}

Fonte - Próprio autor, 2016.

\section{CoNCLUSÃo}

Tal como foi conceituado, a plataforma BIM apresentou uma ampla gama de ferramentas para o setor de projetos civis, possibilitando diversas vantagens para os processos de design e inovações para facilitar a obtenção de documentos atrelados aos projetos. Através do uso do modelo paramétrico gerado pelo BIM, identifica-se uma nova forma de elaboração e desenvolvimento dos projetos que facilita tanto para o desenvolvedor quanto para o gestor dos processos.

A plataforma ainda de maior uso atualmente - O CAD se apresentou como inferior ao BIM, disponibilizando ferramentas e opções mais arcaicas e pouco ágeis considerando as necessidades atuais de eficiência nos processos; além de proporcionar uma visualização mais pobre sobre as interferências comuns ao ato de projetar.

Considerando os dados analisados sobre o que é a Gestão de projetos e sobre a importância da profissão do gestor no mercado contemporâneo, conclui-se que as competências do profissional de gerenciamento estão em concordância com as necessidades atuais do ramo de projetos para construção civil. A agilidade nos processos de confecção do design atrelada a produtos finais de maior qualidade é o diferencial para o sucesso das empresas.

O desenvolvimento das pesquisas apresentadas permitiu concluir que o BIM pode ser uma das soluções para muitas das adversidades vistas na gestão de projetos de construção civil. A plataforma apresentou uma ampla gama de soluções de problemas utilizando de ferramentas novas em comparação ao CAD. Apesar da complexidade de toda uma nova forma de projetar, os processos são simplificados e novos, em sua maioria, na integração com a elaboração das edificações. Além da facilidade de trabalho para todos os colaboradores, tal otimizador de processos de planejamento pode proporcionar grandes economias à empresa por reduzir os problemas e as tomadas de decisões no canteiro de obra. 


\section{REFERÊNCIAS}

ASSAF, A.. Finanças corporativas e valor, 5 ed. São Paulo: Atlas, 2010.

BERNSTEIN, P. G. Barriers to the adoption of building information modeling in the building industry. Autodesk Building Solutions. White Paper, 2004.

CAMPOS, L. F. R.. Gestão de Projetos. Instituto Federal de Educação, Ciência e Tecnologia. CuritibaPR. 2012.

CASTELAN, J.; MILANEZ, A.; FRITZEN, D. Aprendizagem em engenharia utilizando métodos analíticos e numéricos. Revista de Ensino de Engenharia, v. 35, n. 2, p. 37-43, 2016.

CATELANI, W.. 10 motivos para evoluir com BIM. 1. Ed. Brasília-DF. Gadioli Cipolla Branding e Comunicação. 2016.

CATELANI, W... Coletânea Implementação do BIM para Construtoras e Incorporadoras v.1. BrasíliaDF. Gadioli Cipolla Branding e Comunicação. 2016.

COELHO, S. B. S.; MATTAR, D. G.; NOVAES, C. C.. Estudo comparativo dos recursos disponíveis em extranet colaborativa de gestão de projetos, com base em sistemas proprietários ou em software livre. In: XI Encontro Nacional de Tecnologia do Ambiente Construído, 2006.

COELHO, P. G. B.. Project Management Knowledge Learning Enviroment: Ambiente Inteligente de Aprendizado para Educação em Gerenciamento de Projetos. Dissertação de Mestrado em Ciência da Computação. Universidade Federal de Pernambuco. Recife, 2005.

COMPESTRINI, T. et al.. Entendendo BIM. 1. Ed. Curitiba, Paraná, Brasil. 2015.

DINSMORE, C.; CAVALIERI, A.. Como se Tornar um Profissional em Gerenciamento de Projetos.

Preparação para Certificação PMP _ Project Management Professional. Rio de Janeiro. QualityMark, 2003.

DINSMORE, P. C. Winning in Business with Enterprise Project Management, New York: AMACOM, 1999.

EASTMAN, C. et al.. BIM Handbook: A Guide to Building Information Modeling for Owners, Managers, Designers, Engineers, and Contractor. New Jersey. John Wiley \& Sons, 2008.
FERREIRA, S. L;. Da engenharia simultânea ao modelo de informações de construção (BIM): construção das ferramentas ao processo de projeto e produção e vice-versa. In: Workshop Brasileiro de Gestão do Processo de Projeto na Construção de Edifícios, Curitiba, 2007.

FLORIO, W. Contribuições do building information modeling no processo de projeto em arquitetura. In: Seminário Tecnologia da Informação e Comunicação na Construção Civil, 2007, Porto Alegre, 2007.

FONSECA, J. J. S. Metodologia da pesquisa científica. Fortaleza: UEC, 2002.

GIL, A. C. Como elaborar projetos de pesquisa. $3^{a}$. ed. São Paulo: Atlas, 2002.

KERZNER, H.. Gestão de Projetos: as melhores práticas, Porto Alegre: Bookman, 2002.

MARQUES, F.; FLORES, P.; SOUTO, A. P. Desenho e Representação Gráfica, Universidade do Minho. Escola de Engenharia. p. 24, 2017. Disponível em: < https://repositorium.sdum.uminho.pt/bitstream/1822/46 985/1/Capitulo\%201.pdf>. Acesso em: 9 jun. 2019.

MCGRAW HILL CONSTRUCTION, Recent SmartMarket BIM Research, 2014. Disponível em: $<$ https://c.ymcdn.com/sites/www.nibs.org/resource/res mgr/BSA/20140108_moa_jones.pdf>. Acesso em: 14 dez. 2016.

MENEZES, L. C. M. Gestão de Projetos, São Paulo: Atlas, 2001.

ROBSON, C. Real world research: A resource for social scientists and practitioner - researchers. 2nd ed. Blackwell Publishing, 2002.

SELETSKY, P.. Questioning the Role of BIM in Architectural education: A Counter-Viewpoint. AECbytes Viewpoint \#27, August 31, 2006.

TZORTZOPOULOS, P.; COOPER, R.. Design management from a contractor's perspective: The need for clarity. Architectural Engineering and Design Management, 2007.

YESSIOS, C. I., Are We Forgetting Design? AECbytes Viewpoint, \#10, 2004. 